\title{
Verification of Surface Dose for Flattening Filter and Flattening Filter Free Beams in Beam-Matched Medical Linear Accelerators
}

\author{
N Sushma ${ }^{1,2 *}$, Shanmukhappa Kaginelli ${ }^{2}$, Sathiaraj Palanivel ${ }^{1}$, K M Ganesh $^{1}$
}

\begin{abstract}
Background: The purpose of this study was to evaluate the surface dose (SD) of 6 and $10 \mathrm{MV}$ flattening filter beam (FF) and flattening filter free (FFF) beam for different square field sizes in three Beam-matched medical linear accelerators using a parallel-plate ionization chamber. Materials and Methods: The experiment was carried out in a phantom composed of $40 \times 40 \mathrm{~cm}^{2}$ solid Water slabs of varying thickness. Further sheets of solid water phantom were added to take readings in the build-up region for both SSD and SAD technique. Surface doses are measured with a PPC-05 chamber and DOSE 1 electrometer, at measurement depth of $1 \mathrm{~mm}$ interval and all results are plotted relative to the dose measured at $\mathrm{D}_{\max }$ for various field sizes. Surface dose readings are therefore reported as relative surface dose. Results: Surface dose increased linearly with field size for both FF and FFF photon beams in all three beam-matched linear accelerators in both SSD and SAD setup. The surface dose of FFF was higher than FF beams in all field sizes. For the given energy the surface dose difference (relative to $10 \mathrm{x} 10 \mathrm{~cm}^{2}$ field size of $6 \mathrm{FF}$ ) between FF and FFF beam was larger for large field size. For $6 \mathrm{FF}$ and $6 \mathrm{FFF}$ beam the surface dose difference for $5 \times 5 \mathrm{~cm}^{2}$ is $-5.27 \%$, and for $30 \times 30 \mathrm{~cm}^{2}$ it is $12.91 \%$. The measured surface dose differences between linear accelerators are not statically significant $(\mathrm{P}>0.989)$. Similarly, the surface dose difference between SSD and SAD setup was also analysed and had no statistical significance $(\mathrm{P}>0.849)$. Conclusion: Study showed that the surface dose difference between beam-matched linear accelerators are insignificant. The surface dose difference between SSD and SAD setup were also found negligible. Most importantly, changing patients between beam-matched linear accelerators will not have any significant changes in surface dose in clinical setup.
\end{abstract}

Keywords: Surface dose- parallel plate chamber- percentage depth dose- source to surface distance

Asian Pac J Cancer Prev, 22 (8), 2577-2585

\section{Introduction}

The ability to deliver the prescribed dose to the patient within a narrow tolerance is crucial to the success of curative intended radiation therapy. Changes in tumour control probability and normal tissue complication probability of $10-20 \%$ and $20-30 \%$ respectively, can result from a $5 \%$ difference in the delivered dosage (Chetty et al., 2007). The fact that megavoltage (MV) photon beams have a skin-sparing effect is well known, but the exact magnitude depends on a variety of treatment parameters. Depending on the doses delivered, the basal skin layer will experience a number of problems ranging from mild (erythema/epilation) to severe (desquamation/necrosis) reactions. According to the ICRP and the ICRU, the skin depth prescribed for realistic dosage measurements is $0.07 \mathrm{~cm}$, which refers to the skin's interface between the epidermis and dermis layers ("Report 24, ICRU" 1976; Venselaar et al., 2001). At the same time, precise doses to targets near the surface (such as for head, neck, and breast treatments) are critical to avoid under dosing. The clinical use of flattening filter-free (FFF) radiotherapy is growing as accelerators such as the Versa HD (Elekta AB, Stockholm, Sweden) and True Beam (Varian Medical Systems, Palo Alto, CA) are introduced. While surface doses for traditional flattening filter beams (FF) have been studied under a variety of conditions(Carl and Vestergaard, 2000; Kim et al., 1998) but FFF beams have a shortage of data. Simple surface dose vs. field size variations in FFF beams have been reported by several authors(Cashmore, 2016; Kragl et al., 2009), but data on beam-matched linacs are lacking. Switching patients among available linacs can be very convenient and desirable in any high-volume/ high-throughput clinical centre. It may be necessary due to various factors such as linac failure, an unexpectedly high patient load etc. Switching patients treated with a higher treatment modality with different linacs, requires necessary careful thought. Having beam-matched linacs will not only 
improve patient care versatility, but also reduce the social and economic impact of system downtime (Sjöström et al., 2009). In our center four beam-matched Elekta ${ }^{\mathrm{TM}}$ linear accelerators were installed, which consists of three Versa HD TM with photon energies (6FF, 10FF, 15FF, 6FFF and $10 \mathrm{FFF})$ and one Infinity ${ }^{\mathrm{TM}}$ (6FF, 10FF and 15FF). All linear accelerators are having Agility TM head equipped with 80 pairs of multi-leaf collimators (MLC) with a width of $5 \mathrm{~mm}$ at the machine isocenter. The linear accelerators are named as LA1, LA2 LA3 and LA4 (numbers are indicating the accelerators). LA1, LA2, and LA3 are Versa $\mathrm{HD}^{\mathrm{TM}}$ and LA4 is InfinityTM. For FFF beams LA3 was used as a reference machine for LA1 and LA2 whereas for FF beam LA4 was the reference for LA1, LA2 and LA3. Many researchers were reported about the performance of beam-matched linear accelerators (Kairn et al., 2015; Bhangle et al., 2011). Gagneur and Ezzell (2013) and $\mathrm{Xu}$ et al., (2019) reported the patient's specific quality assurance of VMAT, SBRT/SRT for beam-matched linear accelerators. To the best of our knowledge, we could not find any study-related to the surface dose measurement for beam-matched linear accelerators.

In our study surface dose and build-up doses on three beam-matched linear accelerators were evaluated for varying field size, energy in both SSD and SAD techniques.

\section{Materials and Methods}

Elekta's photon beam factory matching allows Percentage Depth Dose at $10 \mathrm{~cm}$ (D10) to be within 1\% for the beam-matched linacs and any averaged point dose (average of the measurements over a $1 \mathrm{~cm}$ range from that point) within the region covering $80 \%$ of full width at half maximum (FWHM) shall be within a $2 \%$ difference as compared to the same points from profiles of other beam-matched linacs for beam profiles of $10 \times 10 \mathrm{~cm} 2$ and 30x30 cm2 field sizes (Sarkar et al., 2013). The D10 of LA4 was kept as reference for FF beams in other linacs viz. LA1, LA 2 and LA 3 within $\pm 1 \%$. Similarly, for FFF beam D10 of LA3 was kept as reference for other two FFF linear accelerators viz. LA 1 and LA 2 within $\pm 1 \%$. Table 1 shows the D10 values for four linear accelerators and percentage difference from the reference linear accelerators.

The surface doses delivered by the FF and FFF beams were measured using a plane-parallel ionization chamber (PPC-05, IBA-Scanditronix, Germany) with DOSE 1 (IBA, Germany) electrometer in a solid water-equivalent phantom with adequate backscattering material. The solid water phantom has a physical density of $1.04 \mathrm{~g} / \mathrm{cm}^{3}$. The PPC05 chamber has a "coin-shaped" sensitive volume with chamber outer diameter of $30.0 \mathrm{~mm}$, height of 14.0 $\mathrm{mm}$, sensitive volume (nominal) of $46.0 \mathrm{~mm}^{3}$, guard ring diameter of $17.8 \mathrm{~mm}$ and guard ring width of $3.4 \mathrm{~mm}$.

Figure 1 shows the experiment setup used to carry out measurement of surface dose in a phantom composed of $40 \times 40 \mathrm{~cm}^{2}$ Solid Water slabs of varying thickness, with 10 $\mathrm{cm}$ of backscatter material to ensure full phantom scatter conditions. Further sheets of solid water phantom were added to take readings in the build-up region for both SSD and SAD technique. Surface doses are measured with a
PPC-05 parallel plate ionization chamber and DOSE 1 electrometer. The chamber was embedded in the $2 \mathrm{~cm}$ slab phantom such that the entrance window of the chamber was flush with the surface with the central axis perpendicular to it. Doses at depth were measured by adding layers of phantom material while maintaining the source to surface distance (SSD) at $90 \mathrm{~cm}$ to the top of the phantom. The effective point of measurement itself was taken as the inside of the entrance window which, for the PPC-05 chamber (with a composite window of $0.1 \mathrm{~mm}$ Mylar) is equivalent to $1 \mathrm{~mm}$ of water. Surface dose measurements therefore represent a measurement depth of $1 \mathrm{~mm}$, and all results are plotted relative to the dose measured at Dmax for the field sizes of $5 \times 5,10 \times 10,15 \times 15,20 \times 20$ and $30 \times 30$ $\mathrm{cm}^{2}$. The measurement was repeated three times for an averaging purpose. Surface dose readings are therefore reported as relative surface dose (RSD) where $\mathrm{RSD}=$ Dsurface /Dmax. As the measurements were performed with PPC 05 chamber, to correct for over-response of chamber detector correction factor (Ci) is applied to the obtained surface dose values(Apipunyasopon et al., 2013). The correction factor " $\mathrm{Ci}$ (L)" is calculated using the empirical relation

$$
\mathrm{Ci}(\mathrm{L})=\mathrm{ai}(\mathrm{L}) 2+\mathrm{bi}(\mathrm{L})+\mathrm{di}
$$

where $\mathrm{Ci}(\mathrm{L})$ is the correction (labelled by an index "i") which is a function of the length of square field's side (L) and ai, bi, and di are arbitrary constants that depend on the type of detector. For parallel plate chamber (Markus type), the constants were $\mathrm{ai}=-0.0006, \mathrm{bi}=0.0314$, and $\mathrm{di}$ $=0.3628$, and the obtained "Ci" values for the field sizes $5 \times 5 \mathrm{~cm}^{2}, 10 \times 10 \mathrm{~cm}^{2}, 15 \times 15 \mathrm{~cm}^{2}, 20 \times 20 \mathrm{~cm}^{2}$, and $30 \times 30$ $\mathrm{cm}^{2}$ were $0.5048,0.6168,0.6988,0.7508$, and 0.7648 , respectively. The procedure was repeated for all three beam-matched Versa HD linear accelerators for surface dose comparison. Data has been analysed for statistical significance using one-way ANOVA.

\section{Results}

Figure 2 depicts differences of the PDD between 6 FF and 6 FFF beams as a function of phantom depth at $1 \mathrm{~mm}$ interval, for five various field sizes $5 \times 5,10 \times 10,15 \times 15$, $20 \times 20$ and $30 \times 30 \mathrm{~cm}^{2}$. The same trend was observed for $10 \mathrm{MV} \mathrm{FF}$ and FFF beams. Figure 3 (a,b,c and d) shows the percentage of surface dose of 6 and $10 \mathrm{MV}(\mathrm{FF}$ andFFF) for SSD and SAD setup for a LA1, LA2 andLA3. From the figure it is very clear that the surface dose increases with the increase in field size. For all the linear accelerators, for both SSD and SAD setup there is a same trend observed in variations of surface dose as a function of field size in Figure 4 ( $a$ and $b$ ). FFF beams shows higher surface dose than FF beams for $5 \times 5$ and $10 \times 10 \mathrm{~cm}^{2}$ field sizes. After $10 \times 10 \mathrm{~cm}^{2}$, it was observed that there was an inverse in the trend, which means the FF beam surface dose was higher than the FFF beams. These trend variations observed for both $6 \mathrm{FF}$ and 10FF beams. The surface dose difference of $6 \mathrm{FF}$ and $6 \mathrm{FFF}$ was less compared to the difference of $10 \mathrm{FF}$ and10FFF (Figure 5). For given energy the surface dose difference (relative to $10 \times 10 \mathrm{~cm}^{2}$ field size of $6 \mathrm{FF}$ ) 
DOI:10.31557/APJCP.2021.22.8.2577

Verification of Surface Dose for FF and FFF photon beams in Beam-Matched Linear Accelerators

Table 1. D10 Values for Four Linear Accelerators and Maximum Difference from the Reference Linear Accelerator

\begin{tabular}{lccccc}
\hline Energy (MV) & LA4 PDD (\%) & LA3 PDD (\%) & LA2 PDD (\%) & LA1 PDD (\%) & Maximum difference \\
\hline $6 \mathrm{FF}$ & 67.42 & 67.74 & 67.53 & 67.13 & -0.47 \\
$10 \mathrm{FF}$ & 72.9 & 72.68 & 72.73 & 72.79 & 0.30 \\
$15 \mathrm{FF}$ & 76.36 & 76.28 & 75.93 & 75.79 & 0.78 \\
$6 \mathrm{FFF}$ & NA & 67.45 & 67.25 & 66.94 & 0.75 \\
$10 \mathrm{FFF}$ & NA & 72.79 & 72.53 & 72.44 & 0.48 \\
\hline
\end{tabular}

between FF and FFF beam was larger for large field size. For $6 \mathrm{FF}$ and $6 \mathrm{FFF}$ beam the surface dose difference for $5 \times 5 \mathrm{~cm}^{2}$ is $-5.27 \%$, and for $30 \times 30 \mathrm{~cm}^{2}$ it is $12.91 \%$. For $10 \mathrm{FF}$ and $10 \mathrm{FFF}$ beam the surface dose difference for $5 \times 5 \mathrm{~cm} 2$ is $-9.8 \%$ ad for $30 \times 30 \mathrm{~cm}^{2}$ it is $21.9 \%$, all percentage difference was calculated relative to $10 \times 10$ $\mathrm{cm}^{2}$ of FF beams.

In Table 2, it was found that the maximum surface dose for 6 FFfor the reference field size of $10 \times 10 \mathrm{~cm}^{2}$ is $32.99 \pm 0.011$ (LA3) in SSD setup and $32.57 \pm 0.006$ (LA1) in SAD setup. For 6FFF beam, the maximum surface dose for $10 \times 10 \mathrm{~cm}^{2}$ is $34.03 \pm 0.007$ (SSD) and $33.37 \pm 0.006(\mathrm{SAD})$ for LA2 and LA1 respectively. For $10 \mathrm{FF}$, the maximum surface dose was $26.86 \pm 0.008$ (SSD) and $26.78 \pm 0.008$ in LA1. For $10 \mathrm{FFF}$ beam the maximum surface dose $28.06 \pm 0.002$ (SSD) and $28.13 \pm 0.007(\mathrm{SAD})$ in LA3 and LA1 respectively. The maximum surface dose in maximum field size $30 \times 30 \mathrm{~cm}^{2}$ for $6 \mathrm{FF}$ is $52.84 \pm 0.011$ (LA1) in SSD setup and 51.68 \pm 0.005 (LA1) in SAD setup. For 6FFF the maximum surface dose was48.90 $\pm 0.022(\mathrm{SSD})$ and $47.98 \pm 0.006$ (SAD) for LA2 and LA1 respectively. For 10FF, the maximum surface dose was $47.76 \pm 0.023$ (SSD) and $47.05 \pm 0.006$ in LA2 and LA1 respectively. For $10 \mathrm{FFF}$ beam the maximum surface dose $41.09 \pm 0.047$ (SSD) and $41.13 \pm 0.008$ (SAD) in LA3 and LA1 respectively.

In Table 2 and 3 , the measured surface dose difference between linear accelerators are not significant statically $(P>0.989)$. Similarly, the surface dose difference between SSD and SAD setup also revealed that they do not have any statistical significance $(\mathrm{P}>0.849)$.

\section{Discussion}

There are three major components which are contributing to surface dose such as head scatter, electron contamination and electron backscatter from the patients. All these factors arises due to beam energy, field size, source to surface distance, beam modifying devices (wedge, compensators and shadow trays) also contribute

Table 2. SD of 6 and 10 MV FF and FFF for Varying Field Sizes for SSD and SAD in All Three Linear Accelerators

\begin{tabular}{|c|c|c|c|c|c|c|c|}
\hline \multirow{3}{*}{$\begin{array}{l}\text { Field size } \\
\left(\mathrm{cm}^{2}\right)\end{array}$} & \multirow{3}{*}{$\begin{array}{l}\text { Energy } \\
(\mathrm{MV})\end{array}$} & \multicolumn{3}{|c|}{ SSD } & \multicolumn{3}{|c|}{$\mathrm{SAD}$} \\
\hline & & LA1 & LA2 & LA3 & LA 1 & LA 2 & LA 3 \\
\hline & & $(\mathrm{SD} \%)$ & $(\mathrm{SD} \%)$ & $(\mathrm{SD} \%)$ & $(\mathrm{SD} \%)$ & $(\mathrm{SD} \%)$ & $(\mathrm{SD} \%)$ \\
\hline \multirow[t]{4}{*}{$5 \times 5$} & $6 \mathrm{FF}$ & $24.24 \pm 0.003$ & $24.70 \pm 0.005$ & $25.31 \pm 0.013$ & $24.68 \pm 0.006$ & $24.44 \pm 0.009$ & $23.42 \pm 0.010$ \\
\hline & $6 \mathrm{FFF}$ & $25.98 \pm 0.007$ & $26.30 \pm 0.013$ & $26.08 \pm 0.012$ & $25.85 \pm 0.006$ & $25.26 \pm 0.010$ & $24.81 \pm 0.008$ \\
\hline & $10 \mathrm{FF}$ & $18.52 \pm 0.012$ & $18.62 \pm 0.003$ & $18.61 \pm 0.005$ & $19.09 \pm 0.007$ & $18.77 \pm 0.029$ & $17.78 \pm 0.009$ \\
\hline & $10 \mathrm{FFF}$ & $21.15 \pm 0.016$ & $21.16 \pm 0.013$ & $21.14 \pm 0.003$ & $21.20 \pm 0.034$ & $20.98 \pm 0.006$ & $20.05 \pm 0.051$ \\
\hline \multirow[t]{4}{*}{$10 \times 10$} & $6 \mathrm{FF}$ & $32.98 \pm 0.011$ & $32.88 \pm 0.010$ & $32.61 \pm 0.104$ & $32.57 \pm 0.006$ & $32.40 \pm 0.008$ & $31.51 \pm 0.009$ \\
\hline & $6 \mathrm{FFF}$ & $33.50 \pm 0.006$ & $34.02 \pm 0.007$ & $33.56 \pm 0.013$ & $33.37 \pm 0.006$ & $33.15 \pm 0.003$ & $32.26 \pm 0.004$ \\
\hline & $10 \mathrm{FF}$ & $26.85 \pm 0.008$ & $26.39 \pm 0.009$ & $26.48 \pm 0.005$ & $26.77 \pm 0.008$ & $26.47 \pm 0.009$ & $25.55 \pm 0.006$ \\
\hline & $10 \mathrm{FFF}$ & $27.89 \pm 0.019$ & $27.98 \pm 0.013$ & $28.05 \pm 0.002$ & $28.13 \pm 0.007$ & $27.83 \pm 0.016$ & $26.91 \pm 0.019$ \\
\hline \multirow[t]{4}{*}{$15 \times 15$} & $6 \mathrm{FF}$ & $40.76 \pm 0.018$ & $39.87 \pm 0.010$ & $40.28 \pm 0.008$ & $40.43 \pm 0.006$ & $40.14 \pm 0.068$ & $40.04 \pm 0.013$ \\
\hline & $6 \mathrm{FFF}$ & $40.17 \pm 0.006$ & $40.82 \pm 0.016$ & $40.19 \pm 0.068$ & $39.97 \pm 0.006$ & $39.83 \pm 0.003$ & $39.45 \pm 0.021$ \\
\hline & $10 \mathrm{FF}$ & $34.30 \pm 0.021$ & $34.01 \pm 0.011$ & $34.23 \pm 0.020$ & $34.74 \pm 0.008$ & $34.47 \pm 0.005$ & $34.19 \pm 0.008$ \\
\hline & $10 \mathrm{FFF}$ & $33.70 \pm 0.021$ & $33.76 \pm 0.014$ & $33.68 \pm 0.038$ & $33.87 \pm 0.008$ & $33.64 \pm 0.019$ & $33.64 \pm 0.010$ \\
\hline \multirow[t]{4}{*}{$20 \times 20$} & $6 \mathrm{FF}$ & $46.62 \pm 0.011$ & $46.64 \pm 0.007$ & $46.46 \pm 0.013$ & $46.56 \pm 0.006$ & $46.05 \pm 0.022$ & $46.11 \pm 0.006$ \\
\hline & $6 \mathrm{FFF}$ & $45.38 \pm 0.008$ & $45.45 \pm 0.008$ & $45.75 \pm 0.016$ & $44.71 \pm 0.006$ & $44.47 \pm 0.012$ & $44.27 \pm 0.007$ \\
\hline & $10 \mathrm{FF}$ & $40.83 \pm 0.023$ & $40.54 \pm 0.005$ & $40.86 \pm 0.011$ & $40.85 \pm 0.007$ & $40.57 \pm 0.007$ & $40.35 \pm 0.008$ \\
\hline & $10 \mathrm{FFF}$ & $38.14 \pm 0.023$ & $37.89 \pm 0.014$ & $38.01 \pm 0.005$ & $38.22 \pm 0.008$ & $38.00 \pm 0.082$ & $37.68 \pm 0.008$ \\
\hline \multirow[t]{4}{*}{$30 \times 30$} & $6 \mathrm{FF}$ & $52.83 \pm 0.011$ & $52.21 \pm 0.024$ & $51.37 \pm 0.001$ & $51.68 \pm 0.005$ & $51.37 \pm 0.019$ & $51.33 \pm 0.005$ \\
\hline & $6 \mathrm{FFF}$ & $48.57 \pm 0.052$ & $48.90 \pm 0.022$ & $48.45 \pm 0.011$ & $47.98 \pm 0.006$ & $47.81 \pm 0.007$ & $47.60 \pm 0.006$ \\
\hline & $10 \mathrm{FF}$ & $46.70 \pm 0.022$ & $46.75 \pm 0.023$ & $46.45 \pm 0.004$ & $47.04 \pm 0.006$ & $46.85 \pm 0.007$ & $46.65 \pm 0.006$ \\
\hline & $10 \mathrm{FFF}$ & $40.82 \pm 0.024$ & $40.73 \pm 0.025$ & $41.09 \pm 0.047$ & $41.12 \pm 0.008$ & $40.87 \pm 0.004$ & $40.65 \pm 0.008$ \\
\hline
\end{tabular}

Values represent Surface Dose \pm Standard deviation; FF, Flattening Filter beam; FFF, Flattening Free Filter beam; LA1,LA2,LA3, Three linear accelerators 

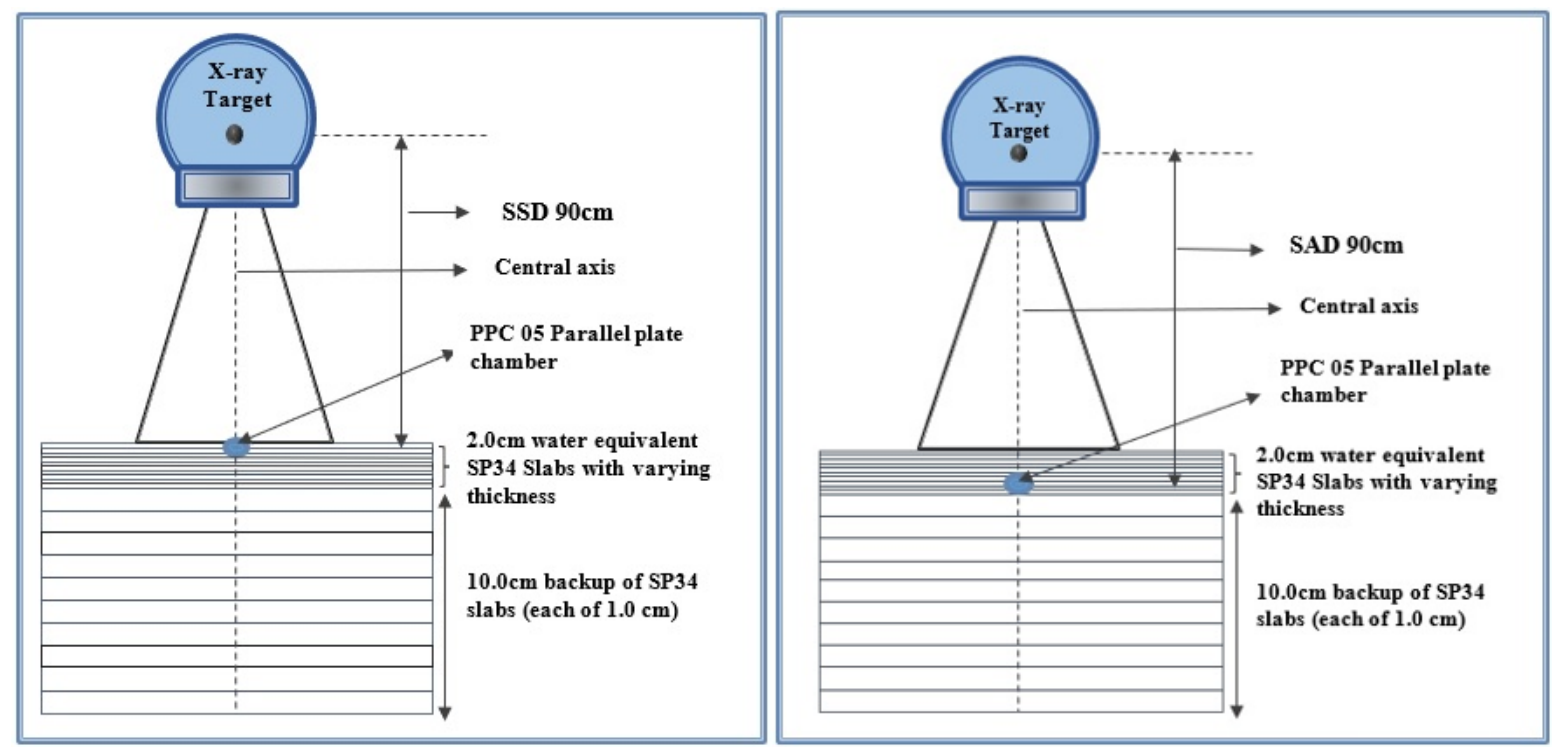

Figure 1. Schematic Diagram of the Measurement Setup Using a Plane-Parallel Markus Chamber and a Stack of Water-Equivalent Solid Phantom Slabs (Varying Thickness) for SSD and SAD Techniques.

to the surface dose (Sigamani et al., 2016). In general, there is no model in treatment planning system accurately accounting for surface dose measurements. Huge difference between surface dose of TPS and measured dose is most commonly observed (Panettieri et al., 2009; Wang et al., 2018). Since, TPS estimation of surface dose is not much accurate and reliable, we need to rely on the physical measurement whenever the clinical situation is demanding. Surface dose measurements is more complex due to the dis-equilibrium behaviour of the beam in the surface/build-up region. As of now extrapolation chamber is the gold standard method for determining the surface reported dose (Reynolds and Higgins, 2015). In addition, with extrapolation chambers the feasibilities of surface dose measurements using other dosimeters such as fixed parallel plate ion chamber, film and cylindrical ion chambers also shown in the literature (Akbas et al., 2016). Eyadeh et al., (2017) used gel dosimeter for surface

Table 3. Difference in SD of 6 and 10 MV FF and FFF for SSD and SAD in All Three Linear Accelerators

\begin{tabular}{|c|c|c|c|c|c|c|c|c|c|c|}
\hline \multirow{2}{*}{$\begin{array}{l}\text { Field Size } \\
\left(\mathrm{cm}^{2}\right)\end{array}$} & \multirow{2}{*}{$\begin{array}{l}\text { Energy } \\
(\mathrm{MV})\end{array}$} & \multicolumn{3}{|c|}{ LA 1} & \multicolumn{3}{|c|}{ LA 2} & \multicolumn{3}{|c|}{ LA 3} \\
\hline & & $\begin{array}{c}\text { SSD } \\
(\mathrm{SD} \%)\end{array}$ & $\begin{array}{c}\text { SAD } \\
(\mathrm{SD} \%)\end{array}$ & Difference & $\begin{array}{c}\mathrm{SSD} \\
(\mathrm{SD} \%)\end{array}$ & $\begin{array}{c}\text { SAD } \\
(\mathrm{SD} \%)\end{array}$ & Difference & $\begin{array}{c}\mathrm{SSD} \\
(\mathrm{SD} \%)\end{array}$ & $\begin{array}{c}\mathrm{SAD} \\
(\mathrm{SD} \%)\end{array}$ & Difference \\
\hline \multirow[t]{4}{*}{$5 \times 5$} & $6 \mathrm{FF}$ & 24.25 & 24.68 & -0.018 & 24.7 & 24.45 & 0.01 & 25.32 & 23.42 & 0.075 \\
\hline & $6 \mathrm{FFF}$ & 25.99 & 25.86 & 0.005 & 26.31 & 25.27 & 0.04 & 26.09 & 24.82 & 0.049 \\
\hline & $10 \mathrm{FF}$ & 18.52 & 19.1 & -0.031 & 18.62 & 18.78 & -0.008 & 18.62 & 17.78 & 0.045 \\
\hline & $10 \mathrm{FFF}$ & 21.16 & 21.21 & -0.002 & 21.16 & 20.99 & 0.008 & 21.14 & 20.05 & 0.052 \\
\hline \multirow[t]{4}{*}{$10 \times 10$} & $6 \mathrm{FF}$ & 32.99 & 32.57 & 0.013 & 32.89 & 32.4 & 0.015 & 32.62 & 31.51 & 0.034 \\
\hline & $6 \mathrm{FFF}$ & 33.5 & 33.37 & 0.004 & 34.03 & 33.15 & 0.026 & 33.57 & 32.26 & 0.039 \\
\hline & $10 \mathrm{FF}$ & 26.86 & 26.78 & 0.003 & 26.4 & 26.47 & -0.003 & 26.48 & 25.55 & 0.035 \\
\hline & $10 \mathrm{FFF}$ & 27.9 & 28.13 & -0.008 & 27.98 & 27.83 & 0.005 & 28.06 & 26.91 & 0.041 \\
\hline \multirow[t]{4}{*}{$15 \times 15$} & $6 \mathrm{FF}$ & 40.77 & 40.43 & 0.008 & 39.88 & 40.15 & -0.007 & 40.29 & 40.04 & 0.006 \\
\hline & $6 \mathrm{FFF}$ & 40.18 & 39.97 & 0.005 & 40.83 & 39.84 & 0.024 & 40.19 & 39.45 & 0.018 \\
\hline & $10 \mathrm{FF}$ & 34.3 & 34.75 & -0.013 & 34.02 & 34.47 & -0.013 & 34.24 & 34.19 & 0.001 \\
\hline & $10 \mathrm{FFF}$ & 33.7 & 33.87 & -0.005 & 33.77 & 33.64 & 0.004 & 33.68 & 33.64 & 0.001 \\
\hline \multirow[t]{4}{*}{$20 \times 20$} & $6 \mathrm{FF}$ & 46.63 & 46.56 & 0.001 & 46.64 & 46.05 & 0.013 & 46.46 & 46.11 & 0.008 \\
\hline & $6 \mathrm{FFF}$ & 45.39 & 44.71 & 0.015 & 45.46 & 44.47 & 0.022 & 45.76 & 44.27 & 0.032 \\
\hline & $10 \mathrm{FF}$ & 40.83 & 40.85 & -0.001 & 40.55 & 40.58 & -0.001 & 40.86 & 40.36 & 0.012 \\
\hline & $10 \mathrm{FFF}$ & 38.15 & 38.22 & -0.002 & 37.89 & 38.01 & -0.003 & 38.01 & 37.68 & 0.009 \\
\hline \multirow[t]{4}{*}{$30 \times 30$} & $6 \mathrm{FF}$ & 52.84 & 51.68 & 0.022 & 52.22 & 51.38 & 0.016 & 51.38 & 51.34 & 0.001 \\
\hline & $6 \mathrm{FFF}$ & 48.58 & 47.98 & 0.012 & 48.9 & 47.81 & 0.022 & 48.46 & 47.6 & 0.018 \\
\hline & $10 \mathrm{FF}$ & 46.71 & 47.05 & -0.007 & 46.76 & 46.86 & -0.002 & 46.46 & 46.66 & -0.004 \\
\hline & $10 \mathrm{FFF}$ & 40.82 & 41.13 & -0.007 & 40.73 & 40.87 & -0.003 & 41.09 & 40.65 & 0.011 \\
\hline
\end{tabular}



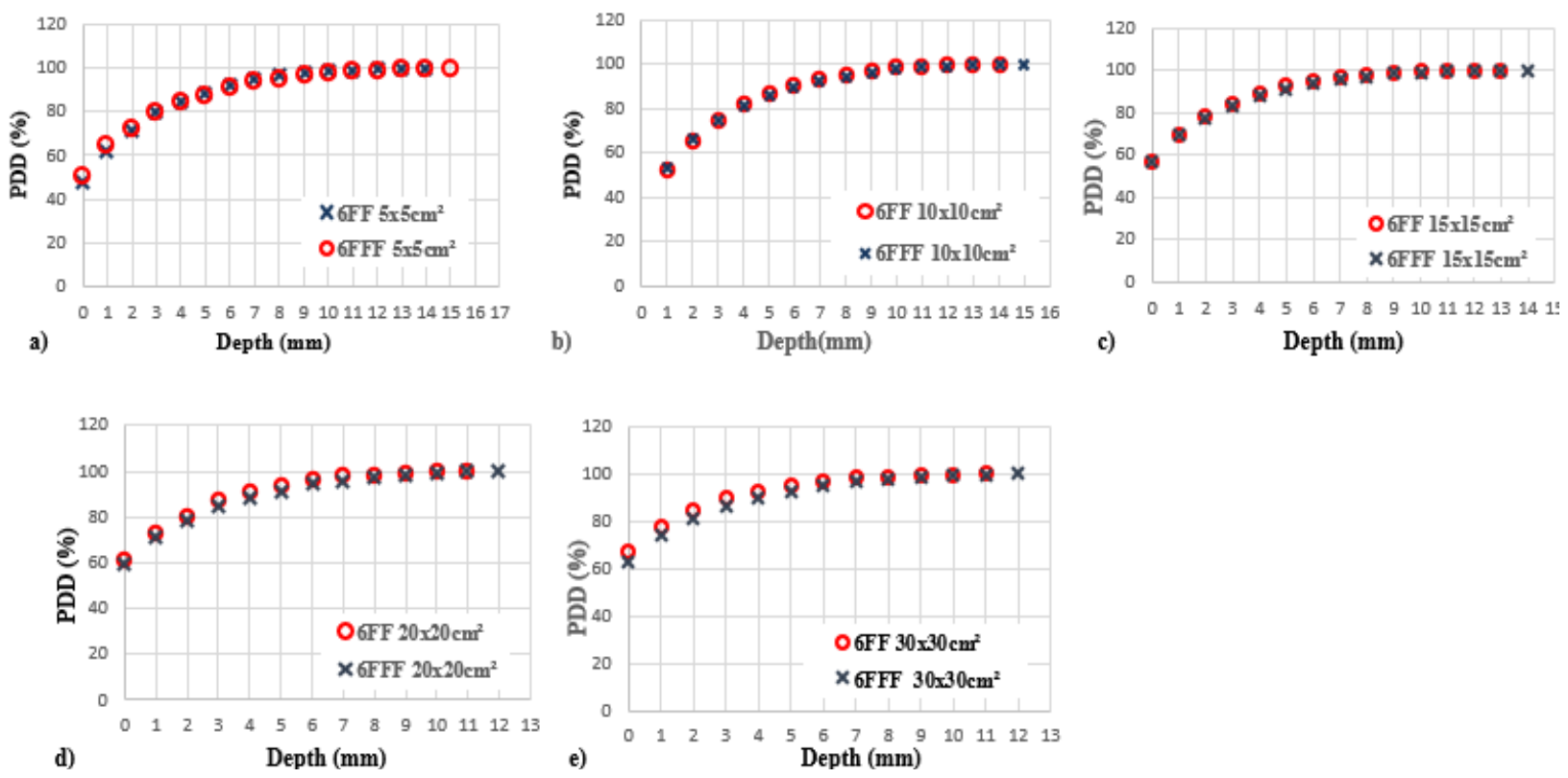

Figure 2. Measured Percentage Depth Dose (PDD) as a Function of the Depth (mm). Comparisons were made between flattening filter (FF) and flattening filter free (FFF) beams with a photon energy of $6 \mathrm{MV}$ and five different field sizes of (a) $5 \times 5 \mathrm{~cm}^{2}$, (b) $10 \times 10 \mathrm{~cm}^{2}$, (c) $15 \times 15 \mathrm{~cm}^{2}$, (d) $20 \times 20 \mathrm{~cm}^{2}$ and (e) $30 \times 30 \mathrm{~cm}^{2}$.

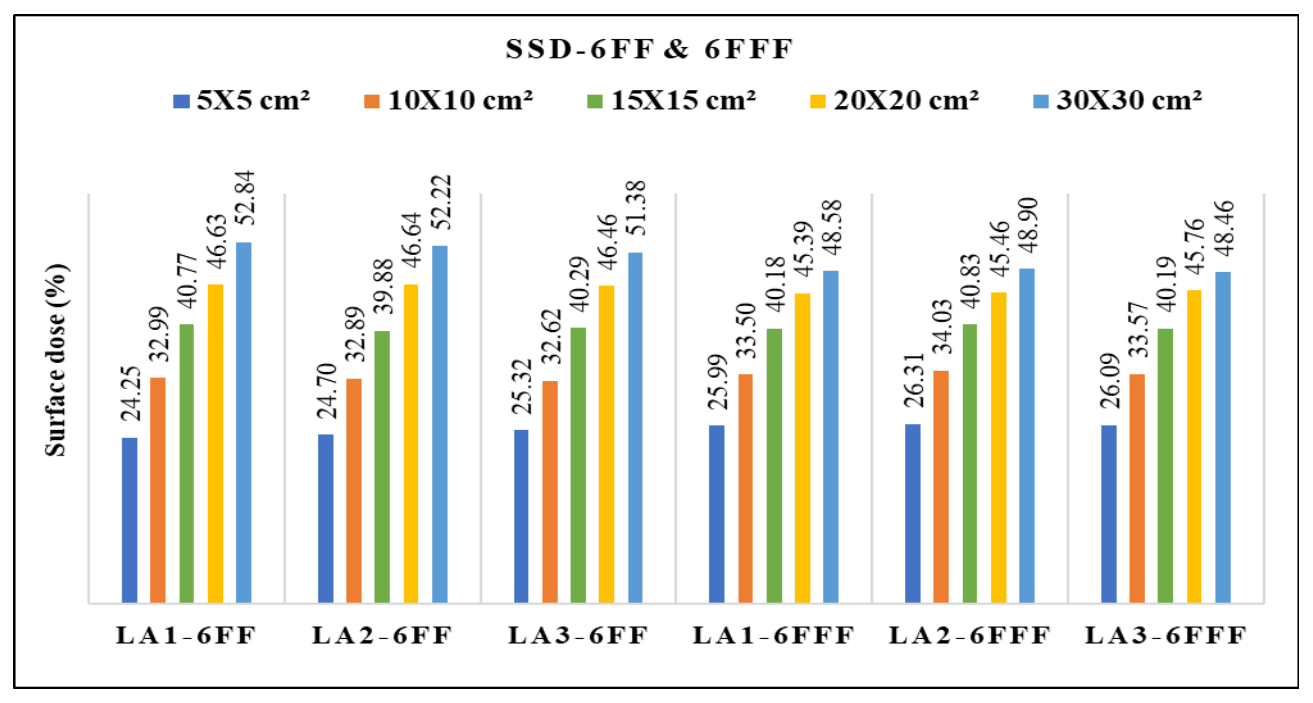

Figure 3a. Variation of Surface Dose for 6 MV FF and FFF Beams Measured with SSD for Three Beam Matched Linear Accelerators

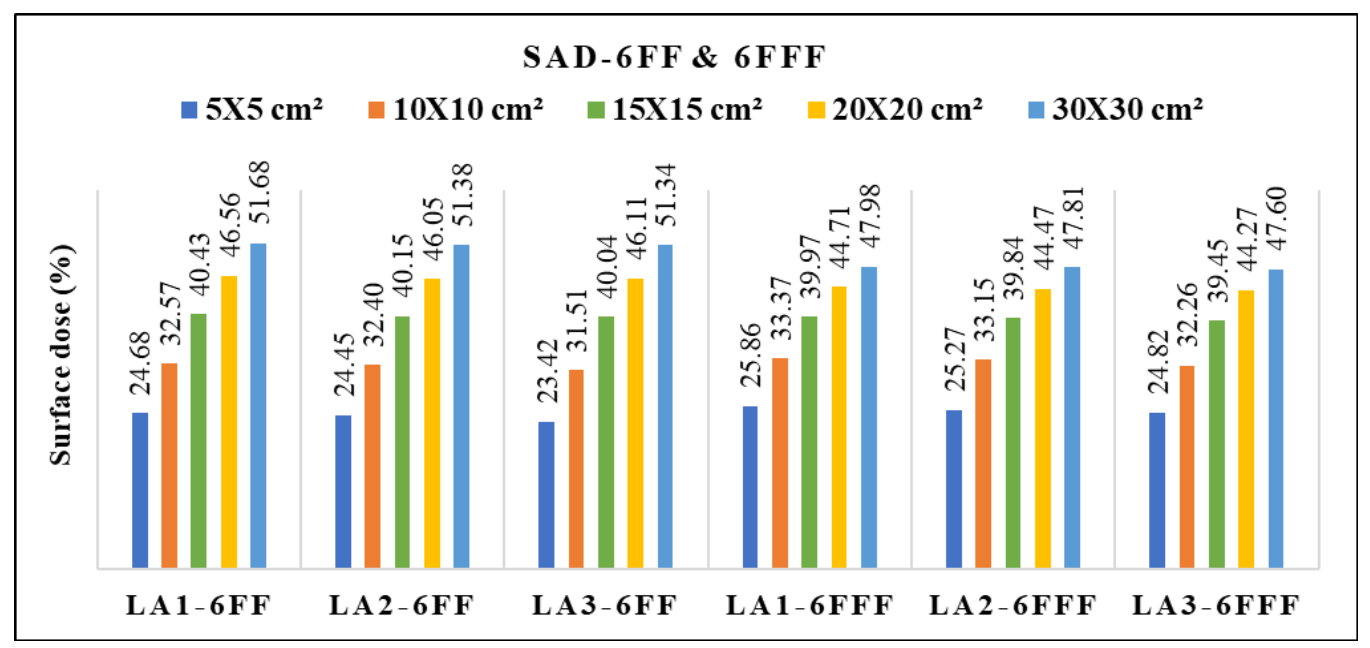

Figure 3b. Variation of Surface Dose for $6 \mathrm{MV}$ FF and FFF Beams Measured with SAD for Three Beam Matched Linear Accelerators 


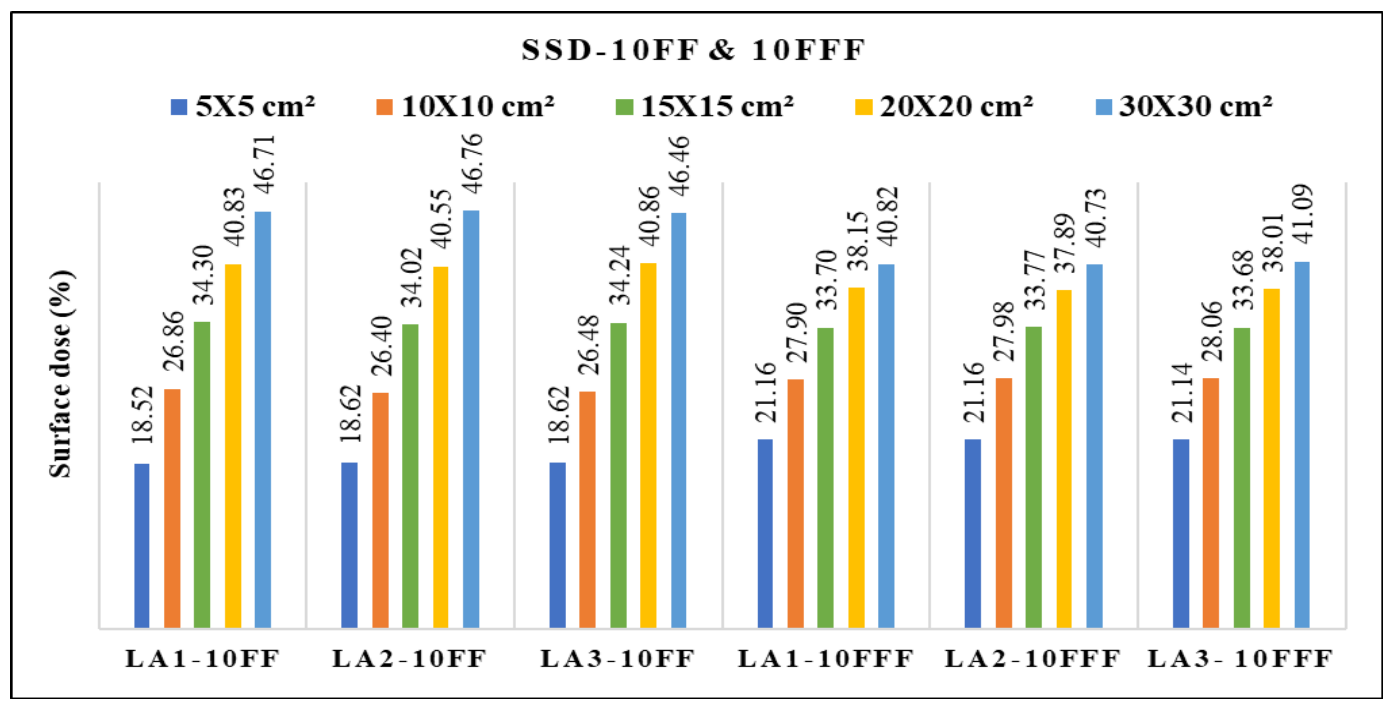

Figure 3c. Variation of Surface Dose for 10 MV FF and FFF Beams Measured with SSD for Three Beam Matched Linear Accelerators

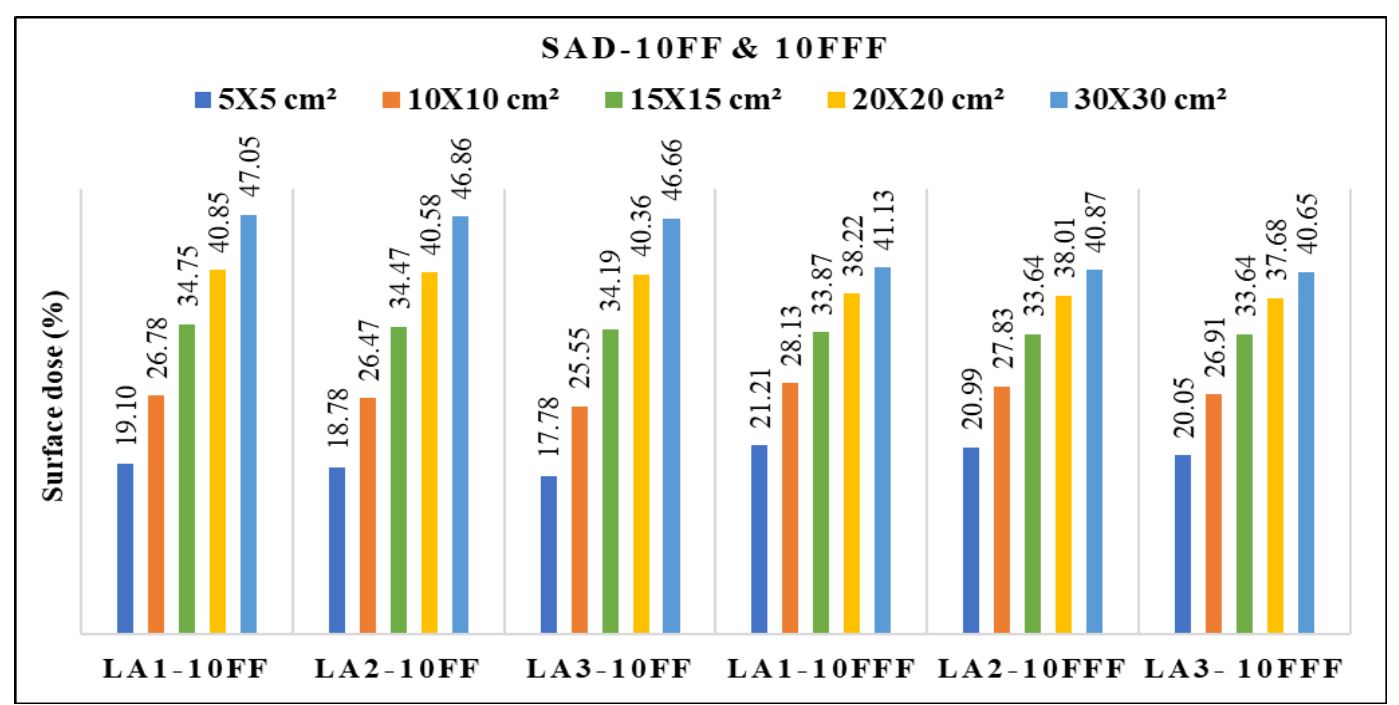

Figure 3d. Variation of Surface Dose for 10 MV FF and FFF Beams Measured with SAD for Three Beam Matched Linear Accelerators

dose measurements, and they compared the results with film, results were comparable. Kinhikar et al., (2009) performed the surface dose measurements using MOSFET and TLD, they showed that inter-fraction deviation of surface dose was within $1.4 \%$. Reynolds and Higgins, (2015) reported about surface dose measurements using TLD, film, OSLD and extrapolation chamber. They compared the dose with extrapolation chamber and they found that the closest match was obtained with the Attix chamber $(-0.1 \%)$, followed by pTLD $(0.5 \%)$, Capintec (4.5\%), Memorial (7.3\%), Markus (10\%), cTLD (11.8\%), eOSL (12.8\%), EBT2 (14\%), EDR2 (14.8\%), and OSL (26\%). The problem associated with fixed parallel plate ion chambers are overresponse in the build-up region. The imbalanced scattering between chamber cavity and medium cause overresponse of the chamber especially in the build-up region, this overresponse can be corrected by applying appropriate correction factors. Gerbi et al., reported the correction factors for correcting the over response of the chamber.

Removing flattening filter increase the dose rate and reduces the extra field scatter. But it increases the low energy components in the spectrum and lead the increment in the surface dose. In this study both FF and FFF beams were analysed for surface dose measurements for different field size. It was observed that the smaller FFF fields were having higher surface dose than FF, beyond $15 \times 15 \mathrm{~cm}^{2}$ the FF surface dose was higher. The same kind of trend have been noticed in the literature Lonski et al., (2017).

From the Figure 3( $a$ and $b$ ) one can understand that the FFF beam gives more surface dose for field size $5 \times 5 \mathrm{~cm}^{2}$ to $15 \times 15 \mathrm{~cm}^{2}$. Beyond $15 \times 15 \mathrm{~cm}^{2}$, FF beam gives higher surface dose than FFF for both 6 and $10 \mathrm{MV}$ energies. This trend may be due to two reasons. Firstly, in a smaller field of FF beam, the collimator settings allow the radiation which is passing through from the central portion of the flattening filter and the concentration of primary radiation is more in such a small field. This primary beam has higher penetration power and this could cause less surface dose. Secondly, when the field size increases, the collimator opening allows more scatter radiation which is originating from the flattening filter (major part), jaws, MLCs, monitor 


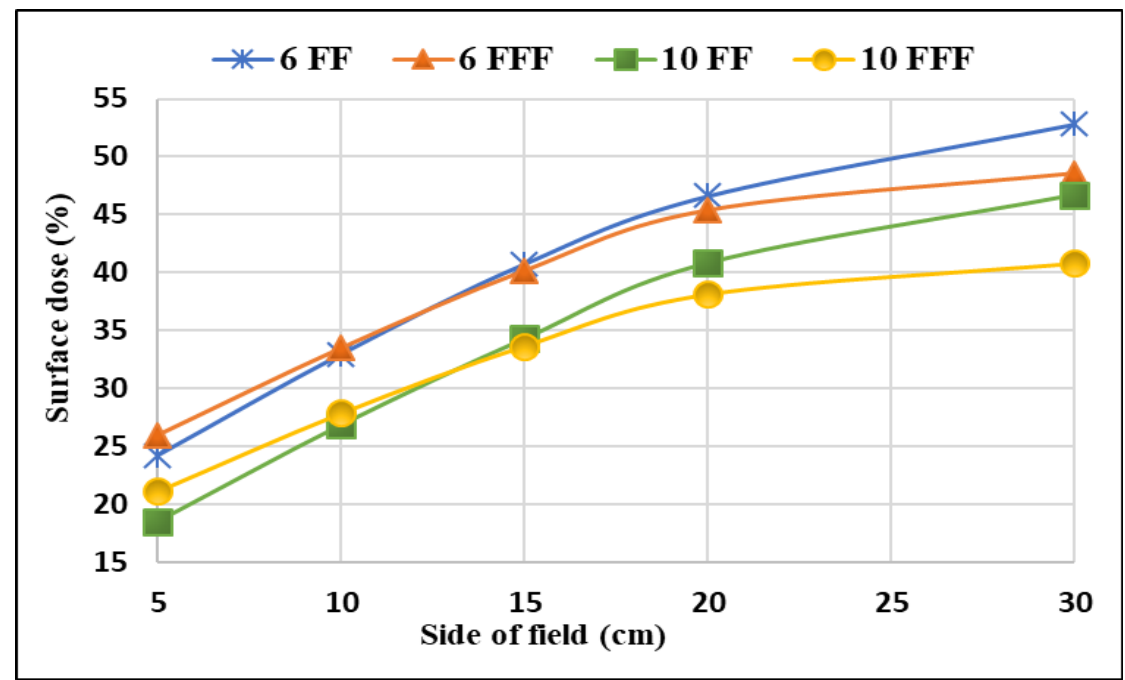

Figure 4a. Variation of Surface Dose with Field Size for 6 and 10 MV FF and FFF Beams (SSD) in LA1

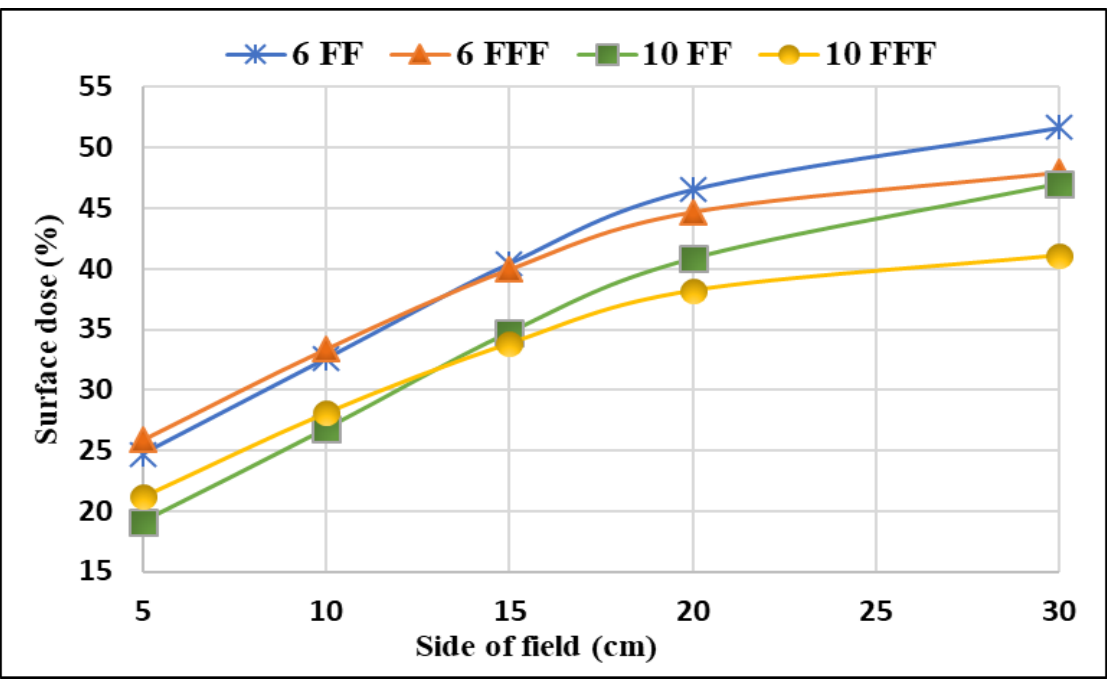

Figure 4b. Variation of Surface Dose with Field Size for 6 and 10 MV FF and FFF Beams (SAD) in LA1

chamber and will mix with the primary component of the beam and reduces the mean energy of radiation which are striking the surface, this softer beam may attribute for an increase in the surface dose. Similarly, in case of FFF beam, the main scattering source of radiation (flattening filter) is absent and the beam may not be harder compared to FF, such a softer FFF beam in a small field causes more surface dose than FF. As the field size increases the

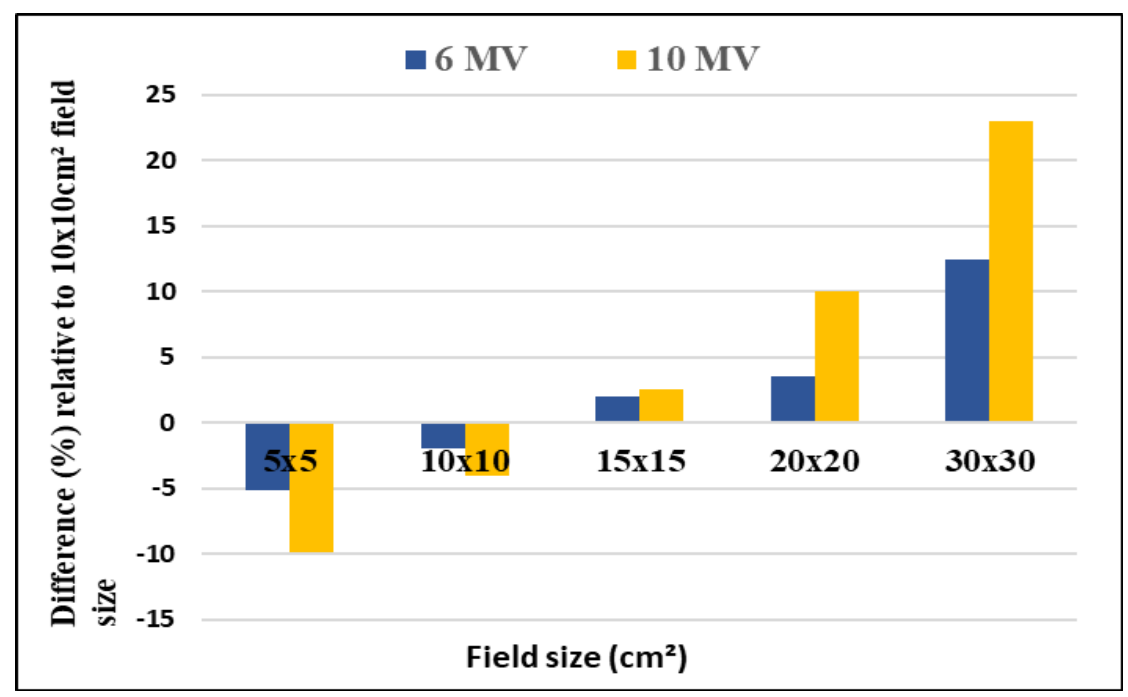

Figure 5. Relative Difference of SD with $10 \times 10 \mathrm{~cm}^{2}$ Field Size for 6 and $10 \mathrm{MV}$ FF Beams 
scattering components in FFF beam may not increase as like in FF, due to less scattering component in FFF, hence it produces less surface dose in larger field sizes. In short, one can interpret that the FF beam is softer than the FFF for larger field size, and for smaller field size FF beam is harder than the FFF beam.

More than one linear accelerator in the institution can be tuned in order to produce same dosimetric quantities called beam-matched linear accelerators. Though, it is beam-matched it should be validated by the physicist before clinical application. This study approached the beam-matched linear accelerators in terms of surface dose measurements, though there is no control over surface dose of beam-matched linear accelerators, it should be verified as a clinical part. Though, linear accelerators are beam-matched, there could be a slight spectral variation between them. This may affect the dosimetric quantities, this study has been done by assuming that the inherent spectral variation cause deviations in surface dose. This study demonstrated that the surface dose is not varying significantly between the beam-matched linear accelerators. Many limitations are there in this study such as, a) except parallel plate ion chamber no other detector are used to confirm the reproducibility of the linear accelerators, b) Surface dose is an important concern in the TBI distance, that is not reported here, c) The results are not compared with any simulated data such as Monte Carlo simulations. Even though the study has above limitations, this is the first study aimed to report the surface dose measurements of beam-matched linear accelerators.

In conclusion, the surface dose measurements have been done for beam-matched linear accelerator for FF and FFF beams in SSD and SAD setup. The FFF beam has higher surface dose up to $15 \times 15 \mathrm{~cm}^{2}$. Beyond $15 \times 15$ $\mathrm{cm}^{2} \mathrm{FF}$ beam has higher surface dose. The surface dose difference between SSD and SAD setup is not significant. Most importantly, study showed that the surface dose difference between beam-matched linear accelerators are insignificant. Changing patients between beam- matched linear accelerators will not have any significant changes in surface dose in clinical setup. This study could be further explored to report the response of surface dose at extended SSD.

\section{Author Contribution Statement}

All the authors have contributed substantially in the study.

\section{Acknowledgements}

\section{Ethical Clearance}

As the study was performed in a phantom and did not involve any humans or human samples or animals, hence the Ethical Clearance was not obtained.

\section{Conflict of interest}

Authors have declared no conflict of interest.

\section{References}

Akbas U, Donmez Kesen N, Koksal C, Bilge H (2016). Surface and buildup region dose measurements with markus parallel-plate ionization chamber, gafchromic EBT3 film, and MOSFET detector for high-energy photon beams. $A d v$. High Energy Phys, 2016.

Apipunyasopon L, Srisatit S, and Phaisangittisakul N (2013). An investigation of the depth dose in the build-up region, and surface dose for a 6-MV therapeutic photon beam: Monte Carlo simulation and measurements. $J$ Radiat Res, 54, 374-82.

Bhangle JR, Narayanan VK, Kumar NK, Vaitheeswaran R (2011). Dosimetric analysis of beam-matching procedure of two similar linear accelerators. J med phys, 36, 176-80.

Carl J, Vestergaard A (2000). Skin damage probabilities using fixation materials in high-energy photon beams. Radiother Oncol, 55, 191-8.

Cashmore J (2016). Surface dose variations in 6 and 10 MV flattened and flattening filter-free (FFF) photon beams. $J$ Appl Clin Med, 17, 293-307.

Chetty IJ, Curran B, Cygler JE, et al (2007). Report of the AAPM Task Group No. 105: Issues associated with clinical implementation of Monte Carlo-based photon and electron external beam treatment planning. Med Phys, 34, 4818-53.

Eyadeh MM, Wierzbicki M, Diamond KR (2017). Measurement of skin surface dose distributions in radiation therapy using poly(vinyl alcohol) cryogel dosimeters. J Appl Clin Med, 18, 153-62.

Gebri B J, khan F M. (1990). Measurement of dose in the buildup region using fixed- separation plane-parallel ionization chambers. Med Phys, 17, 17-26.

Gagneur J, Ezzell G (2013). WE-E-141-02: An improvement in IMRT QA results and beam matching in LINACs using statistical process control. Med Phys, 40, 491.

Kairn T, Asena A, Charles PH, et al (2015). Field size consistency of nominally matched linacs. Australas Phys Eng Sci Med, 38, 289-97.

Kim S, Liu CR, Zhu TC, Palta JR (1998). Photon beam skin dose analyses for different clinical setups. Med Phys, 25, 860-66.

Kinhikar RA, Murthy V, Goel V, et al (2009). Skin dose measurements using MOSFET and TLD for head and neck patients treated with tomotherapy. Appl Radiat Isot, 67, 1683-85.

Kragl G, af Wettersted S, Knäusl B, et al (2009). Dosimetric characteristics of 6 and $10 \mathrm{MV}$ unflattened photon beams. Radiother Oncol., 93, 141-6.

Lonski P, Ramachandran P, Franich R, Kron T (2017). Surface dose measurements in and out of field: Implications for breast radiotherapy with megavoltage photon beams. Z Med Phys, 27, 318-23.

Panettieri V, Barsoum P, Westermark M, Brualla L, Lax I (2009). AAA and PBC calculation accuracy in the surface build-up region in tangential beam treatments. Phantom and breast case study with the Monte Carlo code PENELOPE. Radiother Oncol, 93, 94-101.

Report 24 (1976). ICRP, os13, 142-54.

Reynolds TA, Higgins P (2015). Surface dose measurements with commonly used detectors: A consistent thickness correction method. J Appl Clin Med, 16, 358-66.

Sarkar B, Manikandan A, Nandy M, et al (2013). A mathematical approach to beam matching. Br J Radiology Suppl, 86.

Sigamani A, Nambiraj A, Yadav G, et al (2016). Surface dose measurements and comparison of unflattened and flattened photon beams. J Med phys, 41, 85-91.

Sjöström D, Bjelkengren U, Ottosson W, Behrens CF (2009). A beam-matching concept for medical linear accelerators. 
Acta Oncolo, 48, 192-200.

Tello VM, Tailor RC, WF Hanson (1995). "How water equivalent are water-equivalent solid materials for output calibration of photon and electron beams. Med Phys, 22, 1177-89.

Venselaar J, Welleweerd H, Mijnheer B. (2001). Tolerances for the accuracy of photon beam dose calculations of treatment planning systems. Radiother Oncol, 60, 191-201.

Wang L, Cmelak AJ, Ding GX (2018). A simple technique to improve calculated skin dose accuracy in a commercial treatment planning system. J appl clin Med phys, 19, 191-7.

Xu Z, Warrell G, Lee S, et al (2019). Assessment of beam-matched linacs quality/accuracy for interchanging SBRT or SRT patients using VMAT without replanning. $J$ appl clin Med phys, 20, 68-75.

\section{(c) (i) (8)}

This work is licensed under a Creative Commons AttributionNon Commercial 4.0 International License. 